Pacific Journal of Mathematics

FINITE GROUPS HAVING AN INVOLUTION CENTRALIZER
WITH A 2-COMPONENT OF TYPE PAL $(3,3)$ 


\title{
FINITE GROUPS HAVING AN INVOLUTION CENTRALIZER WITH A 2-COMPONENT OF TYPE PSL $(3,3)$
}

\author{
MORTON E. HARRIS
}

\begin{abstract}
A finite group $L$ is said to be quasisimple if $L=L^{\prime}$ and $L / Z(L)$ is simple and is said to be 2-quasisimple if $L=$ $L^{\prime}$ and $L / O(L)$ is quasisimple. Let $G$ denote a finite group. Then $E(G)$ is the subgroup of $G$ generated by all subnormal quasisimple subgroups of $G$ and $F^{*}(G)=E(G) F(G)$ where $F(G)$ is the Fitting subgroup of $G$. Also a subnormal quasisimple subgroup of $G$ is called a component of $G$ and a subnormal 2-quasisimple subgroup of $G$ is called a 2-component of $G$.
\end{abstract}

We can now state the main result of this paper:

TheOREM A. Let $G$ be a finite group with $F^{*}(G)$ simple. Assume that $G$ contains an involution $t$ such that $H=C_{G}(t)$ possesses a 2-component $L$ with $L / O(L) \cong \operatorname{PSL}(3,3)$ and such that $C_{H}(L / O(L))$ has cyclic Sylow 2-subgroups. Then $\left|F^{*}(G)\right|_{2} \leqq 2^{10}$.

In order to state an important consequence of Theorem A, we require two more definitions. A subgroup $K$ of a finite group $G$ is said to be tightly embedded (in $G$ ) if $|K|$ is even and $\left|K \cap K^{g}\right|$ is odd for every $g \in G-N_{G}(K)$. A quasisimple subgroup $L$ of a finite group $G$ is said to be standard (in $G$ ) if $\left[L, L^{g}\right] \neq 1$ for all $g \in G, C_{G}(L)$ is tightly embedded in $G$ and $N_{G}(L)=N_{G}\left(C_{G}(L)\right)$.

THeORem B. Let $G$ be a finite group with $O(G)=1$ and containing a standard subgroup $L$ with $L \cong$ PSL $(3,3)$. Then either $L \leqq G$ or $L \neq\left\langle L^{G}\right\rangle=F^{*}(G)$ and one of the following five conditions hold:

(a) $F^{*}(G) \cong \operatorname{PSL}(3,9)$;

(b) $F^{*}(G) \cong$ PSL $(4,3)$;

(c) $F^{*}(G) \cong \operatorname{PSL}(5,3)$;

(d) $F^{*}(G) \cong \operatorname{PSp}(6,3)$;

(e) $F^{*}(G)=H_{1} \times H_{2}$ with $H_{1} \cong H_{2} \cong L$ and $C_{G}(L)=\langle t\rangle$ where $t$ is an involution such that $H_{1}^{t}=H_{2}$ and $L=\left\langle h_{1} h_{1}^{t} \mid h_{1} \in H_{1}\right\rangle$.

Note that Theorem B is a step toward the verification of Hypothesis $\theta^{*}$ of [13] and is therefore of import for completing a proof of the Unbalanced Group Conjecture and the $B(G)$-Conjecture and for completing an inductive characterization of all Chevalley groups over finite fields of characteristic 3 (cf. [13, §1]). Also by applying 
[13, Lemma 2.9], [3, Theorem], [1, Corollary II], [8, Theorem 5.4.10 (ii) I, [3, Table 1] and [6, Tables 3 and 4], it suffices, in proving Theorem $\mathrm{B}$, to assume, in addition to $O(G)=1$, that $L \neq F^{*}(G)=$ $\left\langle L^{G}\right\rangle, F^{*}(G)$ is simple and that $C_{G}(L)$ has cyclic Sylow 2-subgroups. But then Theorem A and the classification of all finite simple groups whose Sylow 2-subgroups have order dividing $2^{10}$ (cf. [4] and [7]) yield Theorem B. Consequently Theorem B is a consequence of Theorem A.

The remainder of this paper is devoted to demonstrating that the analysis of [12] and [14] can be applied to prove Theorem A.

All groups in this paper are finite. Our notation is standard and tends to follow the notation of [8], [12] and [14]. In particular, if $X$ is a (finite) group, then $S(X)$ denotes the solvable radical of $X, O^{2}(X)$ is the subgroup of $X$ generated by all elements of $X$ of odd order and is consequently the intersection of all normal subgroups $Y$ of $X$ such that $X / Y$ is a 2-group and $\mathscr{E}(X)$ denotes the set of elementary abelian 2-subgroups of $X$. Also, if $n$ is a positive integer, then $\mathscr{F}_{n}(X)$ denotes the set of elementary abelian 2-subgroups of order $n$ of $X$. Finally $m_{2}(X)$ denotes the maximal rank of the elements of $\mathscr{E}(X), r_{2}(X)$ denotes the minimal integer $k$ such that every 2-subgroup of $X$ can be generated by $k$ elements and if $Y \subseteq X$, then $\mathscr{f}(Y)$ denotes the set of involutions contained in $Y$.

Clearly, if $X$ is a group, then $m_{2}(X) \leqq r_{2}(X)$ and $r_{2}(X) \leqq r_{2}(Y)+$ $r_{2}(X / Y)$ for every normal subgroup $Y$ of $X$.

2. A proof of Theorem A. Throughout the remainder of this paper, we shall let $G, t, H$ and $L$ be as in the hypotheses of Theorem $\mathrm{A}$ and we shall assume that $\left|F^{*}(G)\right|_{2}>2^{10}$.

Then [9, Main Theorem], [15, Four Generator Theorem], [3, Table 1], [6, Tables 3 and 4] and [2] imply that $4<r_{2}\left(F^{*}(G)\right) \leqq r_{2}(G)$ and that Sylow 2-subgroups of $G$ and $F^{*}(G)$ contain normal elementary abelian subgroups of order 8 .

Clearly $C_{H}(L / O(L))$ has a normal 2-complement by [8, Theorem 7.6.1], every 2-component $K$ of $H$ with $K \neq L$ lies in $C_{H}(L / O(L))$ and $O(H) \leqq C_{H I}(L / O(L))$ (cf. [10, $\left.\S 2\right]$ ). Thus $L$ is the unique 2-component of $H, L$ char $H, S(H) \cap L=O(L)$ and $S(H)=C_{H}(L / O(L))$ by [10, Lemma 2.3].

Since $H / S(H)$ is isomorphic to a subgroup of Aut (PSL $(3,3))$ with $(L S(H)) / S(H)$ corresponding to $\mathscr{F} n n(\mathrm{PSL}(3,3))$ and since $\mid$ Aut $(\operatorname{PSL}(3,3)) / \mathscr{I} n n$ (PSL $(3,3)) \mid=2$, we have $|H /(S(H) L)| \leqq 2$ and $H^{(\infty)}=L$.

Let $S \in \operatorname{Syl}_{2}(H)$ and $T=S \cap L . \quad$ Then $T \triangleleft S, T \in \operatorname{Syl}_{2}(L),|T|=2^{4}$, $T$ is semidihedral and $T=\langle\lambda, y|| y|=8| y|=$,2 and $\left.\lambda^{y}=\lambda^{3}\right\rangle$ for suitable elements $\lambda, y$ of $T$. Also $\Phi(T)=T^{\prime}=\left\langle\lambda^{2}\right\rangle \cong Z_{4}$ and $\Omega_{1}\left(T^{\prime}\right)=$ 
$Z(T)=\langle z\rangle$ for an involution $z$ of $T$. Also $D=\left\langle\lambda^{2}, y\right\rangle \cong D_{8}, Q=$ $\left\langle\lambda^{2}, \lambda y\right\rangle \cong Q_{8}$ and $\langle\lambda\rangle \cong Z_{8}$ are the three distinct maximal subgroups of $T$. Let $P=S \cap S(H)$. Then $P \leqq S, P$ is cyclic, $P \cap T=1$ and $\Omega_{1}(P)=\langle t\rangle$. Also $\mathscr{J}(L)=z^{L}, C_{L / O(L)}(z) \cong \mathrm{GL}(2,3)$ and $S(H)=O(H) P$.

Since $r_{2}(S) \leqq 1+r_{2}(S / P) \leqq 2+r_{2}(T)=4$, we have $S \notin \operatorname{Syl}_{2}(G)$.

LEMMA 2.1. The following four conditions hold:

(a) $|H /(S(H) L)|=2$ and $H / S(H) \cong \operatorname{Aut~(PSL~}(3,3)$ );

(b) there is an involution $u \in S-(P \times T)$ such that $D=C_{T}(u) \in$ $\operatorname{Syl}_{2}\left(C_{L}(u)\right), \quad L\langle u\rangle / O(L) \cong \operatorname{Aut}(\operatorname{PSL}(3,3)), \quad \mathscr{J}(u L)=u^{L}, \quad C_{L / O(L)}(u)=$ $\left(O(L) C_{L}(u)\right) / O(L), O\left(C_{L}(u)\right)=O(L) \cap C_{L}(u), C_{L}(u) / O\left(C_{L}(u)\right) \cong$ PGL $(2,3)$, $O^{2}\left(C_{G}(\langle t, u\rangle)\right) / O\left(C_{G}(\langle t, u\rangle)\right) \cong \operatorname{PSL}(2,3), S=(P \times T)\langle u\rangle, \lambda^{u}=\lambda z \quad$ and $\left.C_{\left.T^{\langle u}\right\rangle}\right\rangle(\langle z, y, u\rangle)=\langle z, y, u\rangle$

(c) $Z(S)=\langle t, z\rangle, P\langle u\rangle$ is dihedral or semidihedral and $S \in$ $\operatorname{Syl}_{2}\left(C_{G}(t, z)\right) ;$ and

(d) $Q=\left\langle\lambda^{2}, \lambda y\right\rangle \in \operatorname{Syl}_{2}\left(O^{2}\left(C_{G}(\langle t, z\rangle)\right), C_{O(H)}(z)=O\left(C_{G}(\langle t, z\rangle)\right)=\right.$ $O\left(O^{2}\left(C_{G}(\langle t, z\rangle)\right)\right)$ and $O^{2}\left(C_{G}(\langle t, z\rangle)\right) / O\left(C_{G}(\langle t, z\rangle)\right) \cong \mathrm{SL}(2,3)$.

Proof. Assume that $H=S(H) L$. Then $S=P \times T$ and $Z(S)=$ $P \times\langle z\rangle$. Since $S \notin \operatorname{Syl}_{2}(G)$, we have $P=\langle t\rangle$. Then $\langle t, y, z\rangle \in$ $\operatorname{Syl}_{2}\left(C_{G}\langle t, y, z\rangle\right)$ and [11, Theorem 2] implies that $r_{2}(G) \leqq 4$. This contradiction implies that (a) holds. For the proofs of (b) and (c) of this lemma, it clearly suffices to assume that $O(H)=1$. Then $P=O_{2}(H)=C_{H}(L), H / P \cong$ Aut (PSL $\left.(3,3)\right)$ and there is an element $v \in S-(P \times T)$ such that $v^{2} \in P, C_{T}(v)=D$ and $C_{L}(v) \cong \sum 4$ by $[6$, Table 4]. Thus $S=(P \times T)\langle v\rangle$. Suppose that $\Omega_{1}(\dot{S}) \leqq P \times T$. Then $\Omega_{1}(S)=\langle t\rangle \times D$ char $S, C_{S}\left(\Omega_{1}(S)\right)=(P \times\langle z\rangle)\langle v\rangle \operatorname{char} S$ and $\langle t\rangle \operatorname{char} S$. Since this is impossible, there is an involution $w \in S-(P \times T)$. Then $L\langle w\rangle \cong$ Aut $(\operatorname{PSL}(3,3))$ since $(T\langle w\rangle) \cap P=1$ and $T\langle w\rangle \epsilon$ $\operatorname{Syl}_{2}(L\langle w\rangle)$. Then, as is well known $\mathscr{J}(w L)=w^{L}$ and there is an involution $u \in T w$ such that $C_{T}(u)=D \in \operatorname{Syl}_{2}\left(C_{L}(u)\right), C_{L}(u) \cong \sum 4, S=$ $(P \times T)\langle u\rangle$ and $C_{T\langle u\rangle}(\langle z, y, u\rangle)=\langle z, y, u\rangle$. Also $u \in N_{G}(\langle\lambda\rangle)$ and $C_{\langle\lambda\rangle}(u)=\left\langle\lambda^{2}\right\rangle$. Thus $\lambda^{u}=\lambda z$ and (b) holds. Hence $Z(T\langle u\rangle)=\langle z\rangle$, $\langle t, z\rangle \leqq Z(S)=C_{P}(u) \times\langle z\rangle$ and (c) holds since $\langle t\rangle$ is not characteristic in $S$. For (d) observe that $C_{G}(\langle t, z\rangle)=C_{H}(z)$ and set $\bar{H}=H / O(H)$. Then $C_{\bar{H}}(\bar{z})=\overline{C_{H}(z)}$ and $\bar{z} \in O^{\prime}(\bar{H})=\bar{L} \cong \operatorname{PSL}(3,3)$. But $O^{2}\left(C_{\bar{H}}(\bar{z})\right)=$ $O^{2}\left(C_{\bar{L}}(\bar{z})\right) \cong \mathrm{SL}(2,3), \bar{Q} \in \operatorname{Syl}_{2}\left(O^{2}\left(C_{\bar{H}}(\bar{z})\right)\right)$ and $O^{2}\left(C_{\bar{H}}(\bar{z})\right)=O^{2}\left(\overline{C_{H}(z)}\right)=$ $\overline{O^{2}\left(C_{H}(z)\right)} \cong \mathrm{SL}(2,3)$. Hence $O(H) Q \leqq O(H) O^{2}\left(C_{H}(z)\right)$,

$$
Q \leqq C_{O(H)}(z) O^{2}\left(C_{H}(z)\right)=O^{2}\left(C_{H}(z)\right),
$$

(d) holds and we are done.

Lemma 2.2. $P=\langle t\rangle, t$ is not a square in $G, S=\langle t\rangle \times(T\langle u\rangle)$, 
$|S|=2^{6}, S^{\prime}=\left\langle\lambda^{2}\right\rangle,\langle z\rangle \leqq N_{G}(S)$ and $t \nsim z$ in $G$.

Proof. Assume that $P \neq\langle t\rangle$ and let $w \in \mathscr{I}(S-Z(S))$. Suppose that $w \in P \times T$. Then $w$ is conjugate in $P \times T$ to an element of $y\langle t\rangle$. Since $C_{S}(y)=C_{S}(y t)=(P \times\langle z, y\rangle)\langle u\rangle$, we have $\Omega_{1}\left(C_{S}(w)^{\prime}\right)=\langle t\rangle$. Suppose that $w \notin P \times T$. Then $C_{P}(w)=\langle t\rangle, C_{S}(w)=\langle t\rangle \times C_{T}(w) \times\langle w\rangle$ and $\Omega_{1}\left(C_{S}(w)^{\prime}\right) \leqq\langle z\rangle$. Since $Z(S)=\langle t, z\rangle$, we have $Q \leqq N_{G}(S)$ by Lemma $2.1(\mathrm{~d}),\langle z\rangle \leqq N_{G}(S)$ and $t^{N_{G}(S)}=t\langle z\rangle$. However $\langle z\rangle \leqq N_{G}(S)$ implies $\langle t\rangle \leqq N_{G}(S)$ and we have a contradiction. Thus $P=\langle t\rangle$ and the lemma is clear.

Since $\mathscr{F}(u L)=u^{L}$, we immediately conclude:

CoROLlaRY 2.3. $\{t, z, t z, u, t u\}$ is a complete set of representatives for the H-conjugacy classes of involutions in $H$. Also $u \mathscr{F}(D) \subseteq u^{H}$.

Note that $T\langle u\rangle=\langle\lambda, y u, u|| y u|=| u|=2,[y u, u]=1,| \lambda|=2^{3}$, $\lambda^{y u}=\lambda^{-1}$ and $\lambda^{u}=\lambda z$ where $\left.z=\lambda^{4}\right\rangle$ and hence [12, Lemma 2.1] lists various facts about $T\langle u\rangle$.

Let $x=\lambda^{2} y$. Then $\mathscr{I}(T)=\mathscr{I}(D)=\{z\} \cup y\langle z\rangle \cup x\langle z\rangle$ and $y\langle z\rangle \cup$ $x\langle z\rangle=y^{T}$. Also $C_{S}(y)=\langle t, u\rangle \times\langle z, y\rangle, C_{S}(x)=\langle t, u\rangle \times\langle z, x\rangle, m_{2}(\langle t\rangle \times$ $T)=3$ and $\mathscr{E}_{8}(\langle t\rangle \times T)=\{\langle t, z, y\rangle,\langle t, z, x\rangle\}$. Hence $m_{2}(S)=4$ and $\mathscr{E}_{16}(S)=\{\langle t, u, z, y\rangle,\langle t, u, z, x\rangle\}$. Note also that $u^{S}=u^{T}=u\langle z\rangle$ and $\exp (S)=8$.

Set $A=\langle t, u, z, y\rangle$ and $B=\langle t, u, z, x\rangle$. Then $\mathscr{E}_{16}(S)=\{A, B\}$, $A \sim B$ via $T,\langle A, B\rangle=\langle t, u\rangle \times D$ char $S, N_{S}(A)=N_{S}(B)=\langle t, u\rangle \times D$, $C_{G}(A)=O\left(C_{G}(A)\right) \times A, C_{G}(B)=O\left(C_{G}(B)\right) \times B$ and $N_{G}(S)=S\left(N_{G}(S) \cap\right.$ $\left.N_{G}(A) \cap N_{G}(B)\right)$.

Let $X=\langle t, u, z\rangle$. Clearly $C_{S}(X)=\langle t, u\rangle \times D$.

Lemma 2.4. $X$ is the unique element $Y$ of $\mathscr{E}(S)$ such that $Y \leqq S$ and $|Y|>4$.

Proof. Let $Y \in \mathscr{E}(S)$ satisfy $Y \leqq S$ and $|Y|>4$. Then we may assume that $Z(S)=\langle t, z\rangle \leqq Y$ and $|Y|=2^{3}$. Then $E_{4} \cong Y \cap(T\langle u\rangle)=$ $\langle z, \tau\rangle$ where $\tau \in \mathscr{I}(T\langle u\rangle)$ and $[\langle\lambda\rangle, \tau] \leqq\langle z\rangle$. This forces $Y \cap(T\langle u\rangle)=$ $\langle z, u\rangle$ and we are done.

Set $M=N_{G}(A)$ and $\bar{M}=M / O(M)$. Clearly $C_{G}(A)=O(M) \times A$ and, interchanging $u$ and $u z$ if necessary, there is a 3-element $\rho \in$ $C_{H}(u) \cap N_{L}(A)$ such that $x$ inverts $\rho, C_{A}(\rho)=\langle t, u\rangle,[A, \rho]=\langle z, y\rangle$ and $\rho^{3} \in O(M)$. Also $C_{\bar{M}}(\bar{t})=\overline{C_{M}(t)}=\bar{A}\langle\bar{\rho}, \bar{x}\rangle=\langle\bar{t}, \bar{u}\rangle \times\langle\bar{y}, \bar{z}, \bar{\rho}, \bar{x}\rangle$ with $\langle\bar{y}, \bar{z}, \bar{\rho}, \bar{x}\rangle \cong \sum 4, C_{\bar{M}}(\bar{A})=\bar{A}$ and $\bar{M} \mid \bar{A} \hookrightarrow \operatorname{Aut}(A) \cong \mathrm{GL}(4,2) \cong A_{8}$. Moreover, it is clear that $O^{2}\left(C_{G}(\langle t, u\rangle)\right)=O\left(C_{G}(\langle t, u\rangle)\right)\langle y, z, \rho\rangle,\langle y, z\rangle \in$ $\operatorname{Syl}_{2}\left(O^{2}\left(C_{G}(\langle t, u\rangle)\right)\right)$ and $O^{2}\left(C_{G}(\langle t, u\rangle)\right) / O\left(C_{G}(\langle t, u\rangle)\right) \cong \operatorname{PSL}(2,3)$. 
LemmA 2.5. $\quad M=N_{G}(A)$ controls the G-fusion of elements in $t^{G} \cap A$.

Proof. Assume that $t^{g} \in A$ for $g \in G$. Let $A<S_{1} \in \operatorname{Syl}_{2}\left(C_{G}\left(t^{g}\right)\right)$. Since $S^{g} \in \operatorname{Syl}_{2}\left(C_{G}\left(t^{g}\right)\right)$, we may assume that $S^{g}=S_{1}$. If $A^{g}=A$, then $g \in M$. Suppose that $A^{g} \neq A$. Then $\mathscr{E}_{16}\left(S_{1}\right)=\left\{A, A^{g}\right\}$ and there is an element $h \in S_{1}$ such that $A^{g h}=A$. Then $g h \in M, t^{g}=t^{g h}$ and the lemma holds.

Let $S \leqq \mathscr{S} \in \operatorname{Syl}_{2}(G)$. Then $S \neq \mathscr{S},|\mathscr{S}|>2^{10}$ and $S<N_{\mathscr{S}}(S)$. Since $Z(S) \leqq N_{G}(S)$ and $\langle z\rangle \leqq N_{G}(S)$, we have $\left|N_{\mathscr{S}}(S) / S\right|=2, t^{N_{S}(S)}=$ $t\langle z\rangle$ and $Z\left(N_{\mathscr{S}}(S)\right)=\langle z\rangle=Z(\mathscr{S})$.

Clearly $O\left(C_{G}(S)\right)=O\left(N_{G}(S)\right) \times\langle t, z\rangle$ and if $\pi$ is an element of odd order of $N_{G}(S)$, then $\pi \in C_{G}(\langle t, z\rangle), \pi \in C_{G}(X), \pi \in C_{G}(\langle t, u\rangle \times D)$ and hence $\pi \in O\left(N_{G}(S)\right)$. Thus $N_{G}(S)=O\left(N_{G}(S)\right) N_{S}(S)$.

As in $[12, \S 4]$, we have $S C N_{5}(\mathscr{S})=\phi$ and there is an element $E \in \mathscr{E}_{8}(\mathscr{S})$ such that $E \leqq \mathscr{S}$. Clearly $z \in E,\left|C_{E}(t)\right| \geqq 4$ and $z \epsilon$ $C_{E}(t) \leqq S=C_{\mathscr{S}}(t)$. Suppose that $\tau \in t^{G} \cap E$. Then $|\mathscr{S}|=\left|\tau^{\mathscr{S}}\right|\left|C_{\mathscr{S}}(\tau)\right| \leqq$ $2^{2} \cdot|S|=2^{8}$. Thus $t^{G} \cap E=\phi, t \notin C_{E}(t),\left|C_{E}(t)\right|=4,\left\langle t, C_{E}(t)\right\rangle=X=$ $\langle t, y, z\rangle,[S, E] \leqq E \cap S=C_{E}(t), N_{\mathscr{S}}(S)=S E$ and $t^{E}=t\langle z\rangle$. Interchanging $u$ and $t u$ if necessary, it follows that we may assume that $C_{E}(t)=\langle u, z\rangle$.

Set $F=\langle y, z\rangle$. Then $A=F \cup t F \cup u F \cup t u F, t F \subseteq t^{G} \cap A, t^{G} \cap$ $(F \cup u F)=\phi$ and $t F \subseteq t^{G} \cap A \subseteq t F \cup t u F$. Consequently:

CoRollary 2.6. Either $t^{M}=t^{G} \cap A=t F$ and $|\bar{M} / \bar{A}|=24$ or $t^{M}=t^{G} \cap A=t F \cup t u F$ and $|\bar{M} / \bar{A}|=48$.

Now the analyses of $[12, \S 5-11]$, with the obvious slight changes, shows that $\left|O^{2}(G)\right|_{2} \leqq 2^{10}$. Since $F^{*}(G) \leqq O^{2}(G)$, our proof of Theorem $\mathrm{A}$ is complete.

\section{REFERENCES}

1. M. Aschbacher, A characterization of Chevalley groups over fields of odd order, Ann. of Math., 106 (1977), 353-398.

2. M. Aschbacher and G. M. Seitz, Involutions in Chevalley groups over fields of even order, Nagoya Math. J., 63 (1976), 1-91.

3. - On groups with a standard component of known type, Osaka J. Math., 13 (1976), 439-482.

4. B. Beisiegel, Über einfache gruppen mit Sylow 2-gruppen der ordnung höchstens $2^{10}$, Comm. in Alg., 5 (1977), 113-170.

5. H. Bender, On groups with Abelian Sylow 2-subgroups, Math. Z., 117 (1970), 164-176.

6. N. Burgoyne and C. Williamson, Centralizers of involutions in Chevalley groups of odd characteristic, unpublished dittoed notes.

7. F. J. Fritz, On centralizers of involutions with components of 2-rank two, I, II, J. Alg., 47 (1977), 323-399. 
8. D. Gorenstein, Finite Groups, Harper and Row, New York, 1968.

9. D. Gorenstein and K. Harada, Finite groups whose 2-subgroups are generated by at most 4-elements, Mem. Amer. Math. Soc. No. 147, Amer, Math. Soc., Providence, R. I., 1974.

10. D. Gorenstein and J. H. Walter, Balance and generation in finite groups, J. Alg., 33 (1975), 224-287.

11. K. Harada, On finite groups having self-centralizing 2-subgroups of small order, J. Alg., 33 (1975), 144-160.

12. M. E. Harris, Finite groups having on involution centralizer with a 2-component of dihedral type, II, Illinois J. Math., 21 (1977), 621-647.

13. M. E. Harris, On PSL $(2, q)$-type 2-components and the unbalanced group conjecture, to appear.

14. M. E. Harris and R. Solomon, Finite groups having an involution centralizer with a 2-component of dihedral type, I, Illinois J. Math., 21 (1977), 575-620.

15. A. MacWilliams, On 2-groups with no normal abelian subgroups of rank 3, and their occurrence as Sylow 2-subgroups of finite simple groups, Trans. Amer. Math. Soc., 150 (1970), 345-408.

Received June 21, 1978. This research was partially supported by a National Science Foundation Grant.

UNIVERSITY OF MINNESOTA

MINNEAPOLIS, MN 55455 


\section{PACIFIC JOURNAL OF MATHEMATICS}

\section{EDITORS}

DONALD BABBITT (Managing Editor)

University of Galifornia

Los Angeles, California 90024

HUGo RossI

University of Utah

Salt Lake City, UT 84112

C. C. MOORE AND ANDREW OGG

University of California

Berkeley, CA 94720

\section{J. DUGUNDJI}

Department of Mathematics University of Southern California Los Angeles, California 90007

R. FINN AND J. MILGRAM Stanford University Stanford, California 94305

ASSOCIATE EDITORS
E. F. BECKENBACH
B. H. NeumanN
F. WoLF
K. YosHIDA

\section{SUPPORTING INSTITUTIONS}

UNIVERSITY OF BRITISH COLUMBIA UNIVERSITY OF SOUTHERN CALIFONIA CALIFORNIA INSTITUTE OF TECHNOLOGY UNIVERSITY OF CALIFORNIA MONTANA STATE UNIVERSITY STANFORD UNIVERSITY UNIVERSITY OF HAWAII UNIVERSITY OF TOKYO UNIVERSITY OF NEVADA, RENO UNIVERSITY OF UTAH NEW MEXICO STATE UNIVERSITY WASHINGTON STATE UNIVERSITY OREGON STATE UNIVERSITY UNIVERSITY OF OREGON UNIVERSITY OF WASHINGTON 


\section{Pacific Journal of Mathematics \\ Vol. 87, No. $1 \quad$ January, 1980}

Spiros Argyros, A decomposition of complete Boolean algebras ..........

Gerald A. Beer, The approximation of upper semicontinuous multifunctions

by step multifunctions . . . . ....................

Ehrhard Behrends and Richard Evans, Multiplicity theory for Boolean

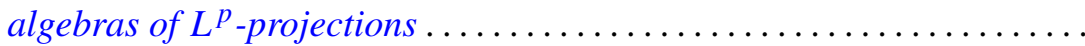

Man-Duen Choi, The full $C^{*}$-algebra of the free group on two

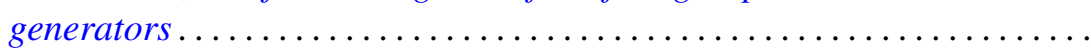

Jen-Chung Chuan, Axioms for closed left ideals in a $C^{*}$-algebra . . . . . . . .

Jo-Ann Deborah Cohen, The strong approximation theorem and locally

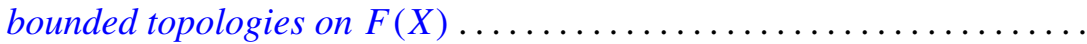

Eugene Harrison Gover and Mark Bernard Ramras, Increasing sequences of Betti numbers............................

Morton Edward Harris, Finite groups having an involution centralizer with

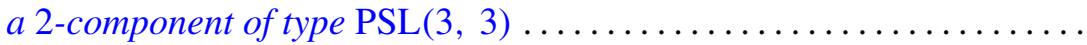

Valéria Botelho de Magalhães Iório, Hopf $C^{*}$-algebras and locally compact

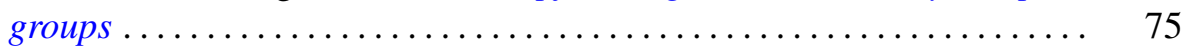

Roy Andrew Johnson, Nearly Borel sets and product measures . . . . . . . . . .

Lowell Edwin Jones, Construction of $Z_{p}$-actions on manifolds . . . . . . . . .

Manuel Lerman and Robert Irving Soare, $d$-simple sets, small sets, and

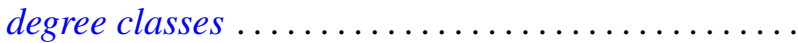

Philip W. McCartney, Neighborly bushes and the Radon-Nikodým property

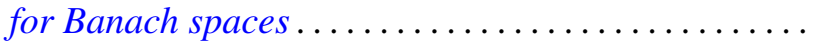

Robert Colman McOwen, Fredholm theory of partial differential equations on complete Riemannian manifolds.

Ernest A. Michael and Carl Preston Pixley, A unified theorem on continuous selections.

Ernest A. Michael, Continuous selections and finite-dimensional sets .

Vassili Nestoridis, Inner functions: noninvariant connected components...

Bun Wong, A maximum principle on Clifford torus and nonexistence of proper holomorphic map from the ball to polydisc.

Steve Wright, Similarity orbits of approximately finite $C^{*}$-algebras . . .

Kenjiro Yanagi, On some fixed point theorems for multivalued

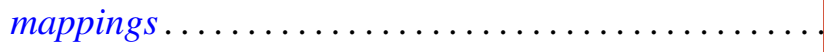

Wieslaw Zelazko, A characterization of LC-nonremovable ideals in commutative Banach algebras 